\title{
A neuropsychological model of free-drawing from memory in constructional apraxia: A theoretical review
}

\author{
Simon McCrea \\ Department of Psychology, University of Regina - Great Plains College, Swift Current, Saskatchewan, Canada S9H 4G3
}

Email address:

Simon.McCrea@uregina.ca

To cite this article:

Simon McCrea. A Neuropsychological Model of Free-Drawing from Memory in Constructional Apraxia: A Theoretical Review. American Journal of Psychiatry and Neuroscience. Vol. 2, No. 5, 2014, pp. 60-75. doi: 10.11648/j.ajpn.20140205.11

\begin{abstract}
Constructional apraxia is an impairment in synthetic activities or abilities such as building, assembling and drawing and it is a major reason for functional disability and for neuropsychological or neurological patients inability to return to work. In this theoretical review a survey of the development of cognitive neuropsychological models of constructional apraxia will be discussed as these relate specifically to drawing abilities. Persons with schizophrenia, somatoform disorders, eating disorders, anosagnosia and right hemisphere disease conditions often present with impairments in the representations or drawings of the body. A plausible cognitive neuropsychological model of constructional apraxia was developed by extracting useful features of previous models and integrating common elements. It is hypothesized that given the relation of the integrity of body-specific representations in the drawings of these neuropsychiatric groups a better understanding of the association between constructional apraxia and free-drawing will provide insights into future clinical and applied neuroscience research.
\end{abstract}

Keywords: Constructional Apraxia, Free-Drawing, Unilateral Cortical Stroke Lesions, Functional MRI

\section{Neuropharmacology and Drawing}

Drawing is an ancient human ability as the discovery of the Chauvet Caves in 1994 in southern France illustrates. A human Venus figure found within the walls of the Chauvet Caves has been dated at approximately 32, 000 years old. These ancient drawings of humans and animals signify the value that human societies have placed on visual artistic abilities for thousands of generations [1]. Free format drawings of houses or dwellings, trees and plant life, and persons are ubiquitous concepts for humanity. These items have a multitude of semantic associates unlike conventional neuropsychological tasks such as clock drawings. Mechanical clocks only have a history of 700 years. Drawings of persons have been used in clinical psychology research since the 1890s to measure intelligence [2] and subsequently to study the unconscious mind and purported psychodynamic processes $[3,4,5]$. There is an extensive history dating from the 1950s associated with the use of human figure drawings in psychiatric experimentation with psychopharmacological agents. Some of this drug research, which involved powerful hallucinogens, was conducted in Saskatchewan, Canada with the goal of developing an experimental model of psychosis. Such basic research studies have been credited, in part, with the eventual elucidation and development of the monoaminergic, and, eventually, the dopaminergic theory, of schizophrenia [6].

Much of this early psychopharmacological research has been forgotten on library shelves, however recent efforts to understand the cognitive neuroscience of human body representations could change that situation. There is renewed interest in studying body representations because of their association with many psychiatric disorders, considerable comorbidity with other more common mental disorders and costs to society. As an example of research in this general direction Schwoebel and Coslett (2005, p. 543) examined 70 stroke patients with an array of neuropsychological tasks and found support for the existence of three types of body representations [7]. In their model the body schema is a dynamic representation of the relative positions of one's body parts derived from multiple sensory inputs, (including proprioceptive), which interacts with the motor system. The body structural description, is a topological map of locations derived primarily from visual input that defines body part boundaries and proximity relationships. Finally the body image is a lexical-semantic representation of the body including body part names, functions, and relations with artifacts. It is presently unknown how these body-specific representations are constructed in the brain, or how these 
representations are related to the apraxias such as in the inability to draw. The apraxias are a general class of neuropsychological motor-based syndromes where the core feature is an ability to perform purposeful planned movements in the absence of a more fundamental primary motor impairment [8]. Drawing then is classed as a particular form of praxis behavior.

Psychometric tests of human figure drawings in particular have been developed to evaluate visuoconstructional abilities across the entire human age span $[9,10]$. Although it is not well known what the neuropsychological correlates of performance on human figure drawing tasks might be, these instruments may be of use to experimenters conducting psychopharmacological research with severe mental illnesses such as anorexia nervosa. Other older research in the psychopharmacological vein has been illuminating on what the neuropsychological correlates of performance on human figure drawings may be.

By way of background, psychoactive drugs such as scopolamine, sodium amytal, mescaline, and lysergic acid diethylamide (LSD-25) began to be explored as potential therapeutic agents in experimental psychiatric research in the 1940s and 1950s [6]. LSD, in particular, was used to study its effects on body image with human figure drawings in healthy participants [11]. Researchers sought to determine whether experimental participants' self-reported body image changes might be manifest in their person drawings when administered LSD. They found that trained clinical psychologists and psychiatrists blind to the effects of the drug (and with no previous experience in the psychodiagnostic use of human figure drawings) were able to reliably discriminate between drawings produced under conditions of mild LSD intoxication and placebo control.

Since the psychopharmacology of many of the hallucinogens such as LSD has now been characterized much better compared to when first used in clinical experimentation in the 1950's, understanding of the functional neuroanatomy associated with intoxication could be invaluable to future researchers. Characteristically, drawings performed under the influence of LSD intoxication had substantially less attention to detail, and greater frequency and severity of distortions - including disunity of the body or dislocation of body parts. Impairments in shape, symmetry, and proportion of drawn figures were also common with LSD intoxication [12]. Savage (1955) described three distinct stages in the process of body image distortions as a consequence of LSD ingestion including (i) an initial subjective pathological accentuation of body image, (ii) progression towards manifold perceptual distortions, and (iii) culmination in dissolution of ego boundaries accompanied by intense feelings of depersonalization. Liebert et al (1958) similarly found that administration of LSD increased the perceived size of the subject's body and its parts without increasing the perceived size of external objects [13].

It would appear, then, that LSD has specific and reliable effects on body image construction in humans, and this research suggests indirect support for the hypothesis that dissolution of the integrity of the body schema may play some role in evolution of pathological disorders such as schizophrenia [14]. Support for these hypotheses is found in a study conducted with administration of $100 \mathrm{mcg}$ of LSD to 21 volunteers. Participants then completed the WechslerBellevue Intelligence Scale during and after intoxication (mean interval $=2$ months) which resulted in a temporary 11 point standard score drop in measured FSIQ $[15,16]$. These researchers attributed this decline to a "loss of the abstract attitude". Judgment was also found to be impaired, however, Object Assembly and Digit Span remained unaffected. Jarvik et al. (1955) similarly showed that at a dosage of $100 \mathrm{mcg}$ healthy controls demonstrated impairments on recall of line drawings of objects (visual memory) as well as recall of verbal paired word associates (auditory memory) [17]. The results of these early studies should not meant be interpreted as suggesting that LSD studies in humans should begin in earnest once again. LSD is a Schedule I drug with no current accepted medical use and it is associated with significant danger to the user.

These caveats aside, only recently have potential models been developed to explain how LSD might affect cortical functioning involved in somesthesis -- largely on the basis of functional neuroimaging studies. Other psychoactive substances such as psilocybin, dimethyltryptamine, and mescaline, have largely been used in the place of LSD in these imaging studies since LSD has remained a strictly controlled substance for over 40 years [18]. However, there are signs of liberalization of pharmaceutical regulatory agencies restriction on the use of hallucinogens for psychiatric research. For example, clinical trials of LSD have recently been conducted by Swiss doctors for the treatment of terminally ill cancer participants [19]. The goal of these studies appears to have been to help terminally ill patients cope with the metaphysical aspects of death. All of these major hallucinogens - which have similar pharmacokinetic properties to LSD - appear to activate the right hemisphere, influence thalamic functioning and increase metabolism in paralimbic structures and in the frontal cortex [18]. Snyder and Reivich (1966) showed that LSD had a propensity towards concentration in monkey brain thalami of an order five times greater than in cortical tissue [20]. Other recent neuroimaging research has examined the neural correlates of dpersonalization previously associated with such experiences as ingestion of LSD. For instance, DeRidder and colleagues (2007) demonstrated that the neural correlates of autoscopy or out-of-body experiences included prominent activation within the right angular-supramarginal junction and the right precuneus (within the right parietal lobes) and subcortically within the posterior thalami [21].

\section{History of the Study Constructional Apraxia}

Conceivably, these body image based perceptions and 
distortions could potentially involve some of the same neural elements associated with the neuropsychological syndrome known as constructional apraxia. Constructional apraxia (CA) has a very general meaning in neuropsychology. Tasks that non-experts might associated with $\mathrm{CA}$ include the Block Design subtest of the Wechsler Adult Intelligence Scale (WAIS) [22], three dimensional cube drawing tasks [23], or stick constructions [24] and various drawing tasks as described by Lezak and colleagues (2004) - [25]. Constructional apraxia has been described as a disturbance that is manifested in "formative activities such as assembling, building, and drawing in which the spatial form of the product proves to be unsuccessful, without there being an apraxia for individual movements" [26]. Roncato et al. (1987) was among the first published studies postulating a plausible cognitive neuropsychological model of CA [27]. Roncato and colleagues' model was based on only three participants studied with the WAIS and the copying tasks of houses, persons, bicycles and cubes. Other clinical neuropsychological tests included the Benton Visual Retention Test [28], Token Test [29], and Warrington and Taylor's Unusual Views Test [30]. Experimental tasks included copying with stimulus objects, copying to verbal command, visual matching, sentence-design matching and synonym judgments. Roncato et al.'s study was conducted on participants in the post-acute phase of stroke on the neurology ward [27].

Roncato et al. (1987, p. 127) purport that copying by drawing has three main processing components [27]. The first component or activity is exploration, which leads to the encoding of a description of the stimulus constituents and their relative relations. The second executive component operates on this formulated description to activate the motor programs after the choice of a scale factor. Scale factors include preparatory activities such as positioning components of a drawing so that these elements will fit on the page. Finally, the matching component is used for comparison of the drawer's production with the stimulus model. Roncato et al.'s first approximation model was exclusively qualitative with little attempt to focus on the precise localization of lesions that would cause the various types of CA. Critically, there was no stepwise articulation or elaboration of the hypothesized cognitive processes involved in the model. The small sample of three participants with limited neuropsychological assessments and no control group further limits generalizability of the findings. Roncato et al. (1987) stated that "...the model presented... has been used mainly for identifying the different sorts of [constructional apraxia] following a description of one or more of the principal processing components. A further step which follows logically requires using the model for analyzing the quality of the errors of constructional apraxia patient's drawings "( $\mathrm{p}$. 127-128) [27].

Constructional apraxia involves building, drawing, and constructing elements into larger gestalts. However, in this theoretical review and accompanying empirical study we will focus entirely on the processes involved in free-drawing rather than on copying or drawing of simple shapes. Free drawing has an equivalent meaning as drawing-from-memory and these terms are often used interchangeably. Although, in recent years, rigorous attempts to characterize the neural correlates of performance on structured constructional tasks such as clock drawing have been undertaken [31]. In this paper free-drawing or the drawing of richly semantically textured complex objects will be examined under standardized conditions with minimal prompts. Arguably, free-drawings have much more ecological validity compared to contrived mechanical clocks. CA has been studied since the early part of the $20^{\text {th }}$ century, however, models of the various processes did not emerge until the 1960's [32]. In the 1980s, cognitive neuropsychology began to gain a better understanding of drawing disorders beyond the simple unilateral focal lesion approach. In the 1990s, compilations of single cases and structural neuroimaging began to highlight specific brain regions hypothesized to be involved in composite drawings. More recently, functional neuroimaging has begun to elucidate the various brain networks involved in drawing-related CA. In this review, the common features of the various theoretical models developed over the last 25 years is elaborated upon with the goal of synthesizing a contemporary model of constructional apraxia.

Table 1. Symptoms of constructional apraxia in left and right hemisphere cortical lesion patients. Note that poor visual acuity, apraxia for single movements, proprioceptive and extrapyramidal disorders must be ruled out for a differential diagnosis of constructional apraxia [66].

\begin{tabular}{ll}
\hline Constructional Apraxia in Left Hemisphere Patients & Constructional Apraxia in Right Hemisphere Patients \\
\hline Reduced size of the figures [71] & Increased number of lines [71] \\
Poor angle representation and incorrect number of angles [72] & Exploded diagrams [72] \\
Motor tremor and right hemiparesis [72] & Individual details accurately represented [72] \\
Poor verbal comprehension [72] & Low Matrices and Block Design scores [72] \\
Impaired object recognition and naming [54] & Failure in association of appropriate colour, shape and size of objects [54] \\
Loss of visual imagery [54] & Loss of spatial relationships [51] \\
Vague description of the appearance of objects [54] & Signs of unilateral left visual neglect and left-sided omissions [51] \\
Oversimplified diagrams with intact global outline [51] & Difficulty in reproducing dimensionality [51] \\
Perseverative errors [51] & A piecemeal copy of the design [51] \\
Comorbidity with dysexecutive syndromes [51] & Tendency to orient drawings diagonally on the page [51] \\
Slow in drawing [66] & Comorbidity with visuospatial agnosia [51] \\
Facilitation by copying [66] & Wrong orientation between component parts [73] \\
\hline
\end{tabular}


Roncato et al. (1987) noted that CA can occur after lesions in either hemisphere [27]. Costa and Vaughan (1962) found a close correlation of drawing-related CA with deficient Block Design and Raven's Matrices [33] scores in right hemisphere lesioned participants [34]. These studies are interesting, yet limited, except from a componential perspective in developing our model since well-structured drawing tasks were invariably used. The associated deficiency between two subtests that now comprise the Perceptual Reasoning Index of the WAIS-IV [35] and CA would suggest a similarly high correlation with nonverbal reasoning. Hence, these initial studies implied that CA would be expected through disruption of the integrated functioning of distributed neural networks in close association with the right hemisphere [36]. Roncato and colleagues noted that drawings in left hemisphere lesioned participants were characteristically coherent with preservation of overall spatial relationships. In contrast, right hemisphere lesioned participants' drawings were often over-simplified with a loss of overall contour [27]. These dual findings of left hemisphere sparsity of features and right hemisphere loss of gestalt were originally hypothesized to result from an under-specified executive deficit in "planning the drawing". Presumably this deficit occurred as a consequence of a disconnection between visuoperceptual and motor processes in the dominant language hemisphere. These visuomotor disconnections were first specified as originating within the left occipitoparietal region [32].

As cognitive neuroscience has evolved, with powerful imaging techniques, notions of hemispheric asymmetries associated with the posterior cortices which build higher order visuospatial percepts have been reconceptualized in much more sophisticated terms of network interactions [37]. These newer models have little resemblance to linear sequential processing models of higher cortical function espoused by theorists such as Luria (1966) [38]. Insight into these dynamic higher-order cognitive functions, associated with the multimodal parietal cortex in particular [39] have been aided by models of cortical interaction in which 'metamodal' brain centers perform particular computational "perceptual-cognitive" operations without the necessity of specific types of sensory input [40]. This means that cognitive operations associated with CA could operate on metamodal schemata that are independent of code type (e.g., visual, auditory, and tactile) although, it might be expected that visuomanual motor-based codes and other metamodal codes (semantic and lexically based codes) would predominate at different stages of network interactions.

Other theory-based models of posterior cortical function, such as the influential two cortical streams view of Milner and Goodale (2006), describe the fundamental throughput pathways that would be expected to instantiate and fundamentally direct such metamodal processing [41].

The dorsal stream consists of two pathways, one in each hemisphere, that transfers spatial location information from the dorsal posterior cortices (occipitoparietal regions) to the superior frontal cortices through the various arcuate and superior fasciculi. The ventral stream also consists of two pathways, one in each hemisphere, that transfers object based information from ventral posterior cortices (occipitotemporal regions) to the inferior frontal cortices through the occipitotemporal, longitudinal, and uncinate fasciculi. These four pathways can synchronize information processing across the hemispheres via commissural fibers and also in the superior/inferior plane via looping U-shaped white matter fibers. Peterson and Rhodes (2003) stated that the posterior aspect of the left hemisphere would instantiate analytic, local, or part-based processing, whereas the posterior aspects of the right hemisphere would be involved in holistic, global, or configural processing [42]. A large body of the functional neuroimaging and lesion research reviewed by Robertson (2004) supports the view that the temporoparietal regions within each hemisphere are chiefly responsible for building up these dual representations [43].

At a more empirical level, drawings may be based on direct visual inputs such as edges, line segments, line drawings, portraits, photographs, or three-dimensional objects that are presented immediately, or that are subsequently removed after short durations.. These types of drawing from copy, after a delay, characterize most of the neuropsychological tasks associated with $\mathrm{CA}$ and used presently. With such brief temporal durations, participants would be expected to rely to a greater extent on visuospatial encoding and memory processes. Alternatively, for the production of facsimiles of real objects, participants may rely on memory retrieval using language and speech based mechanisms that evoke longer term visual representations [44]. Casagrande (2010) hypothesized that such visual representations may be conjured up from the subconscious during hypnogogic imagery or during rapid eye movement related dreaming and sleep as is described in the account of how James Watson visualized the DNA helix [45]. However, is there a seamless and contiguous appearance to these selfgenerated mental images? van Sommers surmised that the notion of imagery as being unified in a single topologically coherent display has not been empirically supported. van Sommers' main support for the view of non-unitary topological mental imagery displays in the mind's eye includes difficulties drawing complex designs (e.g., impossible objects such as the knot design) even when using simple and didactic line-by-line copying strategies [44].

In an attempt to answer such fundamental questions as to the part-based versus seamless nature of imagery, Kosslyn et al. (1985) found that the neural specificity of dimensionally simple unilaterally-presented visual field perceptual tasks were separable from complex imagery tasks in patients whose corpus callosum had been severed [46]. The complex imagery tasks preferentially recruited the left hemisphere, with the right hemisphere having specific difficulty generating multi-part images. Corballis and Sergent (1988) similarly undertook studies of simple and complex imagery tasks in callosally-sectioned patients. These investigators 
found that static imagery tasks employing size or angle partbased descriptions were represented within the left hemisphere, whereas mental rotation of the gestalt aspect of objects was strongly localized to the right hemisphere [47]. These two viewpoints are not necessarily contradictory. Mental rotation might be essential in developing the formative position-independent view of an object and aiding in compositional processes in both structural and descriptive terms. These findings could be congruent with the hypothesis of a strong role for both hemispheres in the overall formative properties presumed to be deficient in fundamental cognitive processes associated with CA.

van Sommers (1989) practically demonstrated aspects of complex mental imagery with drawings of common real objects [44]. van Sommers studied neurologically normal participants' representations of bicycles as only one instance of objects with a rich structure and function knowledge. This knowledge is logically encoded and inter-related verbally within semantics. In these early studies it was shown that participants undertook, (what appeared to be), experimentally directed step-wise refinements of their own bicycle drawings, presumably via access to their hierarchically structured semantic knowledge bases. van Sommers discovered examples of the necessity of access to such essential semantic knowledge bases when drawing. Through task decomposition, van Sommers demonstrated that the drawers must have appreciated the nuances of strictly propositional forms such as "a front wheel of a bicycle must be free to pivot" through sequential approximations in their sketches. The functional and structural knowledge that was encoded propositionally then could augment perceptually-based visuoconstructional processes occurring during various iterations of the drawing plan.

van Sommers (1989) provided the second generation model of CA [44]. His model of graphic processing associated with drawing and copying incorporates Marr's (1982) influential concept of the 2D, $2 \frac{1 / 2}{\mathrm{D}}$ and 3D model of object recognition [48]. van Sommers' model also incorporates long-term memories and representations of familiar visual materials and concepts (Figure 1). Additionally, there are two routes by which stimuli could conceivably access this processing architecture - either through lexical processes/verbal semantics, or through visual input. According to van Sommers, the first stage of the graphic output system is "depiction." Depiction is synonymous with higher-order decisions, such as the type of object to be drawn, the object's orientation, the viewpoint, the level of detail, and the type of boundary that the drawer must determine before beginning. Depiction decisions are non-essential when copying however these must be explicitly implemented when free-drawing from verbal instruction. Production strategy is equivalent to chunking large and small aspects of a figure which are usually arranged hierarchically when drawing. A good example of this chunking stage is the hierarchical decomposition of the Rey Complex Figure. The hierarchical decomposition of this meaningless design is an approach often used when participants systematically attempt to encode both the gestalt and sequential aspects of it [49]. Sequential processes may be incorporated into line-by-line copying processes when grids on the stimulus object and paper are used to systematically map two-dimensionally the segments comprising the drawing plan.

Contingent planning is akin to micro-based problem solving. In contingent planning, the reason for ordering of sequences is primarily geometric degrees of freedom in the drawing plane of a piece of paper - (as in the example of designs that are difficult to draw in the usual orientation of top-left to top-right). Articulation/economy together consist of eight basic executive skills and constraints involved in routine planning. Articulation involves the appropriate starting position, stroke direction, order, and circular schematics, whereas economies involve paper contact, geometric grouping, anchoring, and routine planning. Finally, in this model the integrity of programming of motor movements of the hand can be tested with simple tasks of tracing. van Sommer's 1989 model of the cognitive neuropsychology of drawing and CA is over 20 years old [44] and has been replaced by newer models based on contemporary theory of action planning and mental imagery. Nonetheless, this model's emphasis on verbal and visual routes to mental imagery, as well as the importance of longterm visual memory's interaction with semantics, has been retained by all subsequent models. van Sommers' theory of drawing-related CA, although influential and heavily influenced by then contemporary models of motor control, had not fully integrated findings from lesion studies and that of mental imagery [50]. Note that poor visual acuity, apraxia for single movements, proprioceptive and extrapyramidal disorders must be ruled out for a differential diagnosis of constructional apraxia [66].

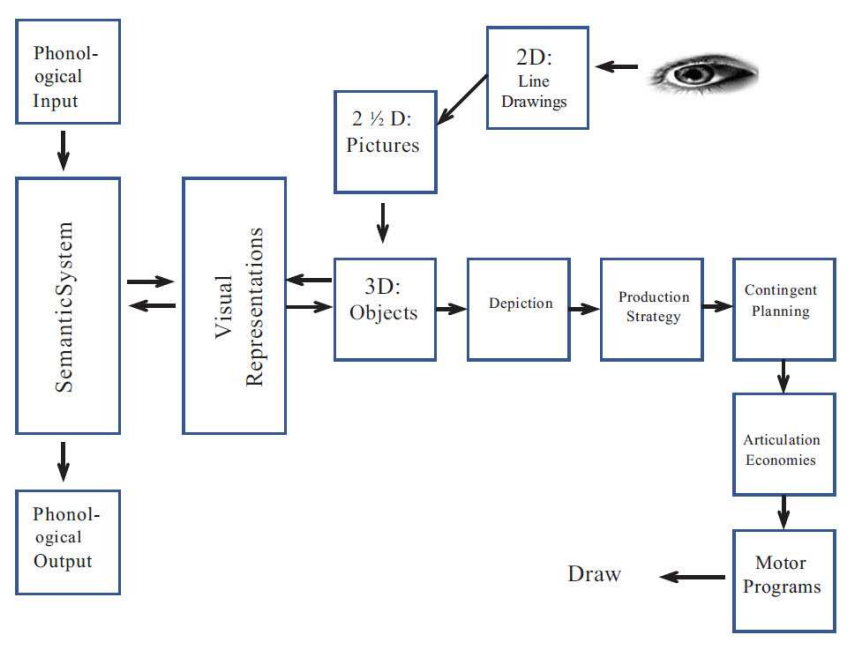

Figure 1. Depiction of van Sommers' model of copying and drawing. Adapted from Figure 22, page 151 in: van Sommers, P. A system for drawing and drawing-related neuropsychology. Cognitive Neuropsychology. 6(2): 117-164. [44]. Reproduced with the permission of Taylor and Francis (C) 1989.

Guerin and colleagues (1999) provided the third major hypothetical model of CA, although these researchers did not articulate a box and arrow diagram [51]. Many of these 
author's contributions were thus conceptual in nature. These authors noted that there had been little progress toward understanding the neural basis and functional interactions of distally located brain systems involved in CA. This was despite the fact that $\mathrm{CA}$ is the most common form of apraxia observed in neuropsychological evaluations [52]. Guerin et al. attributed the limited progress in understanding CA to its inherent complexity, as well as its less striking manifestations compared to other disorders of higher cortical function (e.g., aphasia or agnosia). Guerin et al. note that familiar routine drawings (e.g., geometric shapes and perhaps clock drawings) tasks are often highly overlearned in a motor action sequencing sense, and thus do not place heavy demands on interactions between the semantic and visual representational systems. Hence, this lack of theoretical progress in understanding CA could also be a function of the fact that simple copying or clock drawing tasks had been almost exclusively used in the past. Continued use of such simple visuospatial tasks might actually be hindering progress in this regard.

To date, there have been widespread limitations in experimental designs examining CA. The use of only qualitative instead of both qualitative and quantitative analyses of errors has continued to be a problem. A lack of group and individualized cognitive neuropsychological designs, as well as a lack of lesion analysis and functional neuroimaging methods, until recently, also hindered progress. In their review article, Guerin and colleagues' (1999) argued that the depiction decision components of van Sommers' (1989) model are not specific to drawing, but rather are part of a much more general visual imagery system [44,51]. Their work has been influenced by Farah's (1984) mental imagery model, which posits that visual representations are recalled from long-term visual memory and brought forth into the visual buffer [50]. Guerin and colleagues noted that Farah's model requires an inspection process to examine the image topologically once the images had been cued into the visual buffer, especially when drawing from memory was required. Inspection has been described as encompassing a top-down hypothesis-testing process with a presumed executive component. Participants with generative deficits cannot draw or describe objects from memory yet they are capable of copying and recognizing visual objects. Whereas participants with visual memory deficits are only able to copy models. Farah's model of visual imagery is not specific to drawingrelated CA, yet it has significantly influenced thinking about drawing abilities [50].

If depiction can be better understood through Farah's visual imagery model [50], then what is to be made of the rest of van Sommers' graphic production pathway to motor output involving CA? [44] Guerin et al. (1999) observed that van Sommers' emphasis on production strategies could alternatively reflect a deficiency in general motor planning [53]. In this scenario, contingent planning would be implemented in unfamiliar drawings (e.g., free-drawings) whereas in familiar drawings co-activation of production schemes in associative memory and motor representations in procedural memory would be sufficient for performance. In Guerin and colleagues' view, van Sommers' model overemphasizes systems that constitute action programming motor subroutines, rather than representational types of encoding necessary for unfamiliar drawing (e.g., freedrawing). Guerin and colleagues' (1999) description of CA involves three sets of systems to instantiate drawing abilities: (i) visual perception, (ii) visual imagery and (iii) graphic production [51].

In Guerin et al.'s (1999) model, visual imagery is not always important in CA since familiar, routine, or overlearned drawings can be implemented with activation of associative or procedural motor memory systems alone [51]. In this sense, routine drawings and simple copying are unlikely to tap the perceptual, cognitive, and motor processes involved in pure forms of CA. Copying tasks are often used in neuropsychology and these types of tasks may not use the same neural mechanisms as free-drawing paradigms such as in original artistic visual works or productions. Difficulty with tasks such as clock drawing, which are often used as single measures of $\mathrm{CA}$, may have little relationship or ecological validity to the types of neuropsychological deficits that limit the functional capacity of brain-injured persons. People view, hear, wind, read, wake up to, and interpret many different types of clocks and time-keeping devices tens of thousands of times in their lifetime. In fact, this common neuropsychological test of constructional apraxia is likely overlearned to the point of being automatic.

\section{Constructional Apraxia and Mental Imagery}

Drawing from memory is generally regarded to be a complex task with visual imagery as a core feature [54]. However, as van Sommers (1989) showed, copying can be undertaken by highly automatized motor subroutines without using mental images [44]. Farah posited that the left posterior cortex was critical for mental imagery and that the inferior occipital regions of this area were specifically involved in imagery deficits [55,56]. Trojano and Grossi (1994) noted that among eleven published comprehensive single cases of patients with mental imagery deficits, the two most regular co-occurring syndromes were visual recognition deficits (agnosia) and naming disorders (anomia). The frequency of optic aphasia, in which subjects are unable to name visually presented objects (yet have no difficulty in naming those objects on tactile presentation or verbal description) has also been shown to be unusually high in patients with mental imagery deficits. These patients had the greatest difficulties either in describing imagined objects or drawing them from memory. Among these participants with mental imagery deficits, $50 \%$ had left unilateral posterior-inferior damage, $43 \%$ had bilateral hemispheric posterior-inferior damage, and one left-handed participant had a right posterior-inferior lesion [54]. 


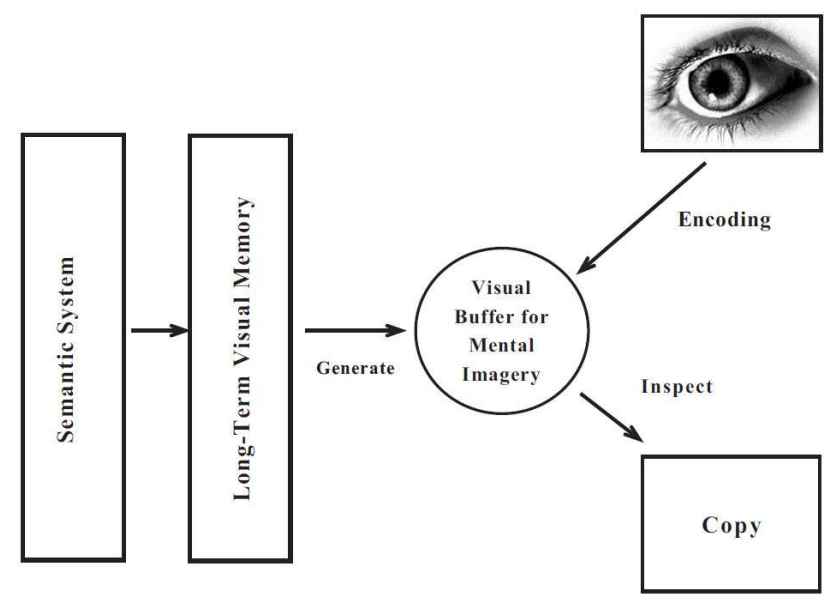

Figure 2. Depiction of Farah's model of mental imagery. Adapted from Figure 12B, page 135 in: van Sommers, P. A system for drawing and drawing-related neuropsychology. Cognitive Neuropsychology. 6(2): 117 164. [44]. Reproduced with the permission of Taylor and Francis (C) 1989. Adapted and redrawn [57] from Figure 1, page 250 in: Farah, M.J. (1985). The neurological basis of mental imagery. In S. Pinker (Ed.). Visual Cognition. Cambridge, MA: MIT Press, pp. 245-271.

Trojano and Grossi (1994) noted that it remains unknown why similar types of lesions do not always elicit similarly corresponding mental imagery deficits, although individual differences in functional localization of cognitive modules might play a role. The finding of regular associations of visual recognition deficits with mental imagery deficits originally led Levine (1978) to propose that there may be redundancy in the primary pathways for visual perception and those of the higher-order perceptual-cognitive process of mental imagery [58]. Thus, empirical review of a number of published cases of agnosia show a tendency for left occipitotemporal lesions to result in visual object agnosia, and right occipitotemporal lesions to result in prosopagnosia or topographical (scene-based) agnosia [42, 59]. Farah (1984) suggested that mental imagery deficits may also be due to loss of access to long-term visual memories, a truism that may be congruent with Levine's hypothesis [50]. Problems in access to long-term memories in this scenario would likely be due to focal lesions in early visual cortex or occipitotemporal cortex. Thus lesions in the left posterior-inferior cortex would result in white matter deafferentiation, and hence blocking of access to the anterior temporal lobe's long-term visual representations. In both Levine and Farah's views there is an assumption of the existence of shared visual recognition and mental imagery perceptual processes.

Aside from studies that have hypothesized left posterior localized neural instantiation of imagery, other studies have examined the relationship between left visual field neglect and mental imagery defects. Left-sided purely "representational neglect" has been demonstrated in a number of participants, beginning with Bisiach and Luzzatti's (1978) famous description of two cases of visualization impairment for landmarks within a well-known piazza in Italy [60]. Their two participants presented with either a right-sided frontal and right temporo-occipital lesion, or a right temporoparietal lesion in which left visual neglect was present in both cases. In such instances, one would naturally presume that mental imagery would still be intact. These two right-hemisphere lesioned participants neglected the left side of the piazza scene and naturally described the right side correctly. However, when a 180 degree change in viewpoint was made the participants were unable to describe the original visual half field which they had accurately described just seconds before! Importantly, at the second viewing, the participants were now able to describe the original left-sided half field neglected in the first trial. If anything, these studies point to the considerable degree that visual representations are coded across both visual fields as well as the potent between-hemisphere integration processes that must occur in real-time during this representational encoding.

Trojano and Grossi (1994) subsequently described eight such cases in the published literature of representational neglect after right hemisphere lesions, and noted that the originators of the representational neglect hypothesis conceived of this as strong proof for a topologically structured representation divided across the two hemispheres [54]. With left neglect, drawings may demonstrate missing left side elements, and therefore collectively such studies imply that the right-sided temporoparietal lesions are apt to adversely affect the building up or structuring of mental representations across both the left and right visual fields. Drawing from memory, however, has been shown to be less sensitive than other tasks in identifying these various types of representational neglect [61]. The reduced sensitivity of drawing from memory to various forms of representational neglect may be due to its complexity and potential for reliance on secondary redundant encoding and lexical and semantic pathways. Alternatively, output processes associated with procedural motor memory, as well as reliance on propositional and logic based processes, may provide additional redundancies. Similarly, whereas participants with visual agnosia have content-specific imagery defects in that they cannot conjure up certain classes of mental images, participants with representational neglect cannot conjure up any mental images at all.

There is, presumably, then a representationally-flexible and isomorphic nature to this system by which mental images are generated, transposed within the visual buffer, and inspected in this process-based framework. A process-based framework of necessity implies some degree of co-operation between distally located brain areas in the complex task of instantiating mental imagery (e.g., see Figure 2). In this scenario, left posterior-inferior sites implicated in mental imagery and right temporoparietal areas involved in dual visual field representational topography constitute a synchronized system. In this process approach, however, content-specific imagery is associated with visual long-term memory. However there are further technical impediments to fully describing and understanding the interactions of the visual imagery and CA systems. Trojano and Grossi stated that mere verbal reports or protocol analysis are heavily reliant on participants' capacity to describe their impairments which are necessarily subjective and prone to error [62]. 
Drawings, by contrast, may have better objectivity in that these verbal report factors can be controlled for and drawings can be qualitatively and quantitatively scored. Similarly, the Block Design subtest of the WAIS, Rey Complex Figure, and Clock-Drawing [63] do not rely on visual and lexical semantics as do free-drawing tasks. Thus, free-drawing tasks could be optimal means of studying constructional apraxia in its full richness and complexity.

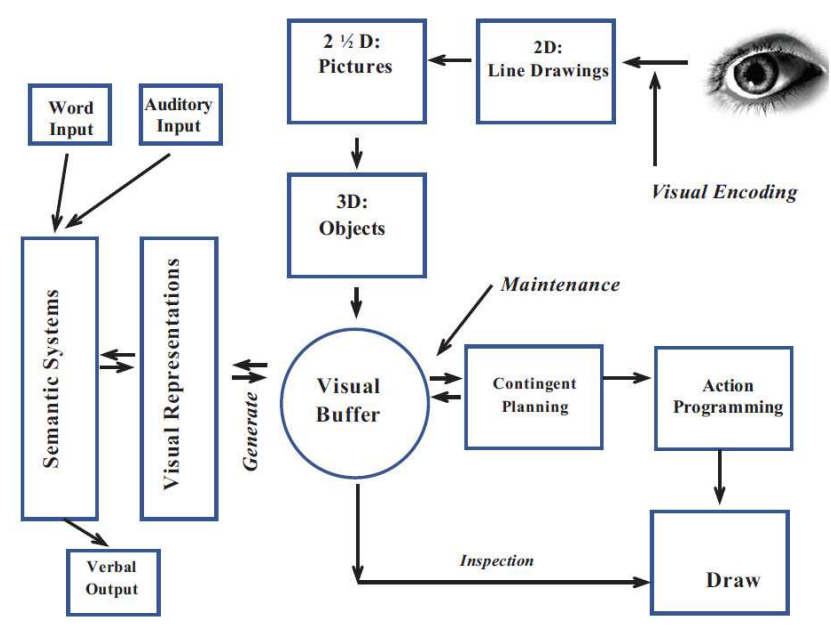

Figure 3. Contemporary model of constructional apraxia-related free drawing. Synthesis of common features of Farah's (1985) [57], Roncato et al. 's (1987) [27], van Sommers' (1989) [44] and Guerin et. al's (1999) [51] model of free-drawing and visual imagery. Note that in this model the graphic production system of van Sommers has been substantially simplified in view of Guerin et al.'s more representational and less motor-based view of drawing abilities. The visual buffer is analogous to a representational medium for mental imagery.

Towards this end, Gainotti et al (1983) found that on mental imagery tasks left-hemisphere lesioned aphasics performed worst, left hemisphere lesioned participants without aphasia performed second worst, right hemisphere participants scored third and non-lesioned participants scored best [64] These researchers' findings strongly suggest a tight link between imagery deficits and reliance on lexical and semantic processes. Further, these results imply that theoretically, mental imagery defects should have a degree of independence from CA. A large study of ideal left and right posterior cerebral artery (PCA) stroke participants (where there is often damage to lateralized occipitotemporal regions exclusively) found that participants with left PCA lesions performed poorly on a pictorial version of the shape and color test [65].

Left temporo-occipital patients (as opposed to left occipital lesions) were selectively impaired on verbal and visual questions. Right PCA patients were only impaired on perceptual tests of shape and colour discrimination. Bilateral PCA lesioned patients were not examined in this study [65].

Collectively, these findings suggest that a wide range of lesions can result in mental imagery deficits pointing towards a distributed model as depicted in Figure 3. It seems based on this evidence that mental imagery is unequivocally related to verbal abilities and semantic knowledge on both (i) functional and (ii) neuroanatomical grounds [54].

\section{Syndromes Associated with Constructional Apraxia}

Dupuy and Godefroy (2007) noted that diagnosis of CA is often made on the basis of copying tasks such as the ClockDrawing test, the copy condition of the Rey Complex Figure (Osterreith, 1944), or three-dimensional block constructions [66]. In assessing CA, these investigators caution clinicians to rule out competing hypotheses as to the origins of $\mathrm{CA}$ involving other perceptual-cognitive mechanisms or damage to elements of a distributed neural network illustrated as in Figure 3. For example, dysexecutive syndromes in particular must be ruled out, since such disorders can adversely affect performance on virtually any complex motor function task [66]. Thus, in theory, a definitive diagnosis of CA can only be made with a comprehensive and individualized neuropsychological assessment. Taken together, CA is largely a diagnosis made on the basis of exclusion. In large samples of patients referred for comprehensive general neuropsychological evaluations, Gainotti (1985) reported a frequency of $\mathrm{CA}$ in unilaterally lesioned hemispheric participants of between $15 \%$ to $40 \%$ [67]. Larger lesions would typically be associated with elevated rates of CA, as would lesions to critical neuroanatomical loci or network connections.

Hemianopia and fluent aphasia are often comorbid symptoms of CA [68]. Dupuy and Godefroy (2007) interpreted this comorbidity as support for their contention that $\mathrm{CA}$ is a posterior cortical syndrome [66]. However, historically the neuropsychological literature is replete with CA cases with damage to prefrontal cortex and with associated executive function impairments. Although not all neuropsychologists agree that $\mathrm{CA}$ is frontal in origin. Among the most common behavioral symptoms of frontal CA is a "closing-in" behavior when participants are actively drawing [69]. A patient with frontal-variant of CA and closing-in behavior would often draw over the actual model when attempting to copy it. Gainotti also demonstrated that diffuse cortical damage was associated with closing-in related CA. Whether this type of behavior is truly symptomatic of CA or is indicative of a frontal-release, poor motor planning, mirror movements or motor artifact is unknown. Recall that frontalrelease symptoms sometimes occur in the instance of frontal damage where brain-lesioned participants exhibit behaviors that would normally be inhibited. Conversely, mirror movements are those that occur when the participant often with a medial frontal lesion mimics the motor movements of the examiner [70].

From a neurodevelopmental perspective closing-in drawing behaviors can also be observed in young children, presumably as a function of the immaturity of myelination within the prefrontal cortex [69]. Proximal closing-in behaviors were observed by Gainotti at an elevated frequency with the presentation of the most complicated of models to be 
copied. Luria and Tsvetkova (1964) demonstrated a frontal variant of CA most often appearing when the most complex of figures were presented [74]. Other studies showed that examiners who provided verbal cues as to the correct production that the subject should make [75] or cues to sequencing [76] nonetheless facilitated performance for this frontal-variant of CA. Collectively, these findings suggest that executive processes such as planning and verification maybe critical components in the motor programming associated with complex drawing. It is unclear if these older conceptions of CA are congruent with contemporary views depicting $\mathrm{CA}$ as a perceptual process heavily-dependent upon posterior cortical functions.

Clock drawings, the drawings associated with the MiniMental Status Examination [77], and even the monolithic Rey Complex Figure task lack definitive links with realworld knowledge of language and lexicosematics - (e.g., however see also Lezak et al.'s, 2004 reference to the housetree-person drawing test and the bicycle test) [25]. These mainstay diagnostic tests of clinical neuropsychology possess little resemblance to the perceptual, cognitive, lexicosemantic, and motor programming requirements of complex drawing tasks used in naturalistic situations. Trojano and Conson (2008) noted that drawing tasks are the most frequently used tests to assess CA [73]. Drawing-related CA tasks include free-drawing or drawing from memory of a named object (e.g., house, tree or person) or simple copying tasks. Trojano and Conson noted that in free-drawing tasks, specific scoring techniques, relevant content analysis procedures, and systematic control for premorbid drawing ability are required for the evaluation of theoretical models of drawing. These controls are rarely introduced in practice.

When visual neglect and severity of lesion are controlled for researchers have found that left and right hemisphere lesioned participants have a similar prevalence of CA $[78,79]$. These findings provide support for the hypothesis of a mutual dependence between the two hemispheres in CA, as well as qualitative differences in processes across the two hemispheres depending on the lesion site. De Renzi (1982) noted that CA is most often associated with parieto-occipital lesions [80] although severity of CA did not differ between frontal and posterior lesion variants [81]. That both frontal and posterior cortical regions appear to be involved in CA alludes to the complexity in the structuring and sequencing of perceptual-cognitive processes in this disorder. Other researchers, notably Kirk and Kertesz (1989), did not find that there was specific intra-hemispheric localization in determining CA [72]. Furthermore, Marshal and colleagues (1994) found that subcortical right anterior lesions were especially apt to cause CA [82]. As such, a modern view might be if frontal regions are involved in $\mathrm{CA}$, patient errors are likely attributable to defective motor programming components (see Figure 3) rather than perceptual encoding and imagery-based processes.

These somewhat contradictory findings are consonant with three conclusions. First, as Dupuy and Godefroy (2007) have emphasized, CA has been consistently underestimated in its degree of complexity [66]. Second, many assessment tasks have been too easy for participants, or researchers have used insufficient numbers and/or types of tests to rule out competing alternative neuropsychological explanations. Third, there have been too few models of the complete picture of CA since van Sommers (1989) detailed attempt at developing a cognitive neuropsychological box model [44]. Even in subsequent models [27,51,57] there has been minimal specification as to what neural correlates correspond to different modules and interactive systems.

The most comprehensive and modern account of CA to date is Grossi's (1991) model [83].

This model specifies that the lexical route works by activation of familiar constructional verbal schemata (e.g., circle) and that a line-by-line procedure predominates in the use of spatial analysis for which there are no extensive preexisting constructional representations (e.g, doodling). Interestingly, line-by-line copying procedures are often observed in certain visual agnosic participants, such as integrative agnosics, who presumably have difficulties accessing the lexical route [42,59]. Complex objects such as houses, trees, and persons, are likely to place heavy demands on lexicosemantic encoding (as opposed to copying simple figures like circles or squares) and presumably would accentuate visual imagery and the distributed free-drawing network. Even familiar objects, which most people have ample experience drawing (e.g., squares and circles) most likely do not involve the lexical route and largely rely on the integrity of procedural memory or a simple constructional schema (e.g., see Figure 4). Trojano and Conson (2008) argued that frontal lesions are not sufficient to produce "true constructional apraxia", and that comprehensive assessment of frontal (e.g., executive function) and posterior functions (e.g., agnosia, receptive language, visuospatial functions etc.) is required for a differential diagnosis [73]. Complex freedrawings with minimal prompts then would likely rely on both a combination of the lexical and heuristic routes.

Comprehensive studies and compilations of single participants using a broad cross-section of neuropsychological instruments will be essential for developing contemporary models. As an example, Trojano and Grossi (1998) described a case of pure CA and emphasized the "need for detailed assessments of visuoperceptual, representational and executive abilities, as well as for analyses of drawing productions, to gain insight into the nature of single participants' disabilities...(p.49) - [84]. The complexity of the multiple distributed perceptual, cognitive, and motor processes involved in CA points to the necessity of using convergent research methodologies. Conventional studies of $\mathrm{CA}$ in diffuse dementing conditions with non-lexically based tasks will likely impede theoretical developments in understanding this neuropsychological disorder. Instead, studies of thoroughly evaluated neuropsychological participants with single focal contiguous stroke lesions demonstrating variants of CA with different lesion topographies could be implemented using computerized psychological experiments. Such comprehensive analysis of single case studies using spatial attention, spatial 
perception, executive function, spatial cognition, and object recognition paradigms might then be able to provide more fine-grained reaction time and accuracy data with which to specify models of $\mathrm{CA}$ in detail. Finally, selected single participants with CA could be studied using structural and functional neuroimaging with which to test various hypotheses associated with the model described herein (see Figure 4).

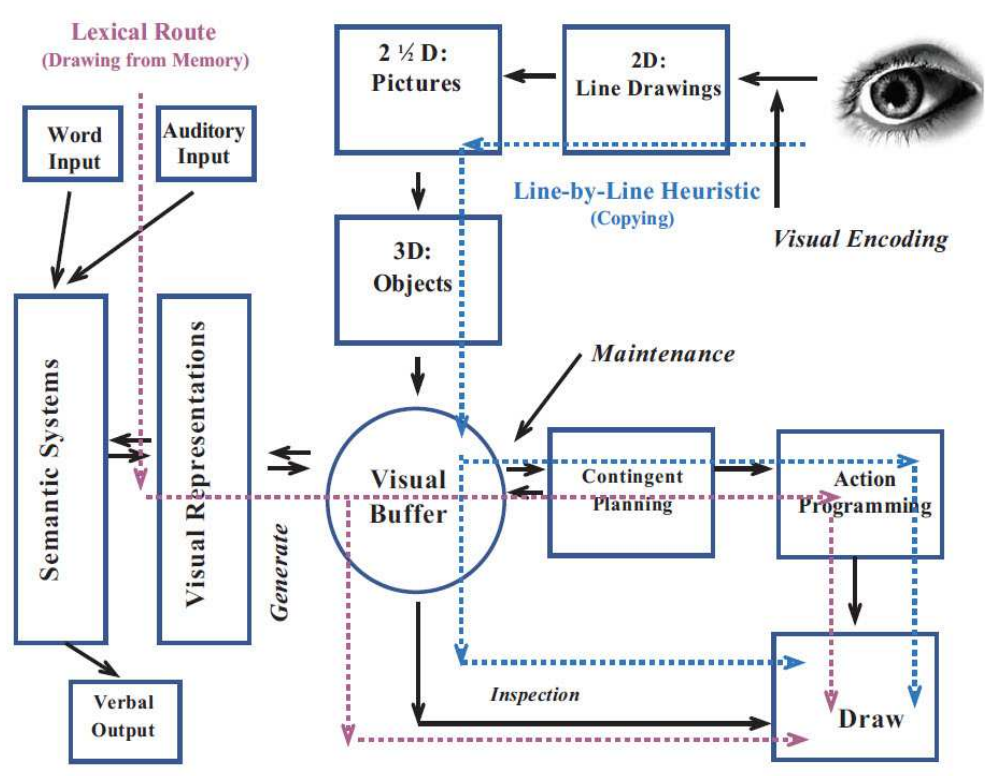

Figure 4. Contemporary model of constructional apraxia-related free drawing incorporating the lexical and line-by-line heuristic routes. Cognitive neuropsychological model illustrating Grossi's (1991) lexical route involved in drawing from memory (purple) and line-by-line heuristic used in copying from a model (blue) [83].

\section{Modeling Cortical Interactions in Free-Drawing}

Functional neuroimaging in combination with plausible models offers a means of examining the neural correlates of free-drawing related CA. Such studies have been aided by the development of functional magnetic resonance imaging (fMRI)-compatible writing tablets [85]. Computational advances, such as structural equation modeling [86], are likely to be essential in understanding $\mathrm{CA}$ in its natural complexity. Neilson (1975) was among the first to question whether CA might be construed of entirely as an interhemispheric disconnection syndrome [71]. In his review, Neilson examined similarities in drawing performances of splenial-lesioned callosal participants using the left and right hands. Studies by Gazzaniga et al. (1965) and LeDoux et al. (1977) relatedly established that split-brain participants demonstrated a left hand advantage on the Block Design subtest of the WAIS $[87,88]$. These types of dual hemispheric models of CA, in addition to subsequent models of imagery $[46,47]$, offer counterpoints to the usual single hemisphere processing accounts of CA.

Alternatively, intra-hemispheric disconnections of left mesial occipital white matter associated with acquired alexia, visual object agnosia, and mental imagery deficits have long been identified [54]. All of these related disconnection studies point towards the critical involvement of large-scale brain systems in free-drawing CA. Kirk and Kertesz (1993) found that both left and right subcortical lesions were significantly associated with impairment on the writing, Block Design, and Raven's Coloured Progressive Matrices [89] tasks of the Western Aphasia Battery [90]. Left subcortical lesions in striatal pathways proved to be particularly detrimental and were associated with numerous signs of CA (see Table 1). However, this study found that neither left nor right subcortical lesions were associated with differential subjective drawing errors. The authors concluded that the lack of differential scoring errors after left or right subcortical lesions suggest that cortical substrates, rather than subcortical substrates, are involved in drawing processes [89].

Laeng (2006) examined CA in left and right hemisphere stroke participants [91]. Laeng found that in a computeradapted task arranging identical items required a coordinated metric representation constructed by the right hemisphere. Laeng also showed that the degree of impairment between specific types of spatial relations used, such as in categorical or coordinate encoding, was highly correlated with the degree of impairment found in constructional tasks such as stick constructions. Laeng noted that up to $58 \%$ of variance in such macro-level constructional tasks (e.g., stick constructions) could be explained by performance patterns on simple scaled-down computerized tests of categorical or coordinate encoding - e.g., see Kosslyn et al, (1992) for a discussion of categorical versus coordinate encoding [92]. To digress, Jager and Postma (2003) summarized categorical representations as representing the general attributes of the spatial structure of a visual stimulus. In contrast, coordinate representations specify precise spatial locations of objects in metrical units. The left hemisphere appears to be specialized for the 
computation of categorical spatial representations while the right hemisphere is specialized for the computation of coordinate ones [93]. Collectively, these recent studies point to the modern view of CA as due to: (i) perceptual-cognitive factors, (ii) predominance of posterior cortical function involvement, and (iii) integrative cognitive processes occurring across hemispheres.

\section{Neuroimaging of Drawing-Related Constructional Apraxia}

Makuuchi et al (2003) examined drawing by copying using fMRI and found activation within the superior parietal lobules and intraparietal sulci, bilaterally [94]. The supramarginal gyrus was activated in the left hemisphere in $29 \%$ of participants, whereas the right supramarginal gyrus was activated in $41 \%$ of participants. Brodmann's area (BA) 39 (angular gyrus) was not activated in participants where the task was to trace an object using the dominant right hand. Incidentally, there was an extended period of right occipitotemporal activation found in the drawing by copying condition by Makuuchi et al. This extended activation was attributed to the temporal operational capacity of the visual imagery buffer's components involved in copying as opposed to simple naming procedures [94].

In another study, Farias and colleagues (2006) compared the effects of cuing by writing and by drawing in individuals with aphasia using a combined lesion and fMRI study [95]. Drawing was found to facilitate verbal responses to a greater degree than writing, which the researchers attributed to richer priming of perceptual, structural, and functional motor aspects of objects during drawing. Moreover, these authors found that it was the motor act of drawing, rather than the quality of the drawing, that was correlated with loci of semantic activation and with subsequent accuracy in the naming of the target object. Activation in the homologues of Broca's area in the right hemisphere, as well as within the left fusiform gyrus and left dorsolateral prefrontal cortex differentiated the drawing versus writing comparisons in fMRI. The left fusiform gyrus has previously been shown to be involved in object knowledge [94] and the left dorsolateral prefrontal cortex in semantic selection [96] and phonological processing [97].

Facilitation by naming in aphasia while drawing thus appears to occur through the widely distributed semantic network with recruitment of intact right hemisphere brain regions probably through mechanisms of commissural fiber lexical access. There are some limitations with these early studies of the functional neuroimaging of drawing. Makuuchi et al.'s (2003) study did not require hand movements and thus the premotor and motor activations could be due entirely to motor imagery or representational processes rather than constituting true manifestations of CA related behavior [94].
The Makuuchi task also involved copying the object (e.g., object in a presented picture) with the index finger without visual feedback, which is contrary to actual drawing tasks that involves initiating and maintaining a flexible grip of a writing instrument in conjunction with visual and proprioceptive feedback. Other studies using advanced fiber optics technologies in the functional neuroimaging environment have come closer to the mark.

Ferber et al (2007) utilized an fMRI-compatible drawing tablet in a task with both proprioceptive and visual feedback [98]. In this experimental design there were two pivotal conditions: (i) to draw a picture from a visually presented word without an accompanying image, or (ii) to draw a copy of a continuously displayed real object. Unlike the Makuuchi (2003) study, the Ferber study was more akin to spontaneous free-drawing in CA as opposed to mere copying. Drawing from a word presentation minus the condition of copying of realistic objects demonstrated activation of the right anterior cingulate, right medial frontal gyrus, right middle frontal gyrus, and right superior parietal lobule. In contrast to lineby-line copying, drawing minus copying of realistic objects would be expected to emphasize the importance of access to the lexical route. In a third condition, copying versus mere tracing was found to activate the anterior cingulate and the medial frontal gyrus probably involved in hierarchical planning and motor control mechanisms [98]. Copying minus drawing also activated the left middle occipital gyrus, left cuneus, and left lingual gyrus (left occipitotemporal regions) which have been shown in lesion studies to be involved in visual imagery [59] and uploading of schemata into the visual buffer. Conceivably then, this online visual imagery could help to ensure that the elements of the drawn copy match the displaced target line drawing.

Ferber and colleagues (2007) posit that drawing-frommemory requires "maintenance of attention, access to memory systems to retrieve information about the stimulus, and internal monitoring of whether the performed action conforms to the original intention...Whereas copying requires constant visual feedback processes and cross modal shifts of attention to compare one's own copy on the page with the model object" (p. 1092) [98]. The visual feedback hypothesis was supported by time-course analyses of regions of interest within the left lingual gyrus and left cuneus, which showed more sustained blood-oxygen level dependent activation in the copy of realistic objects. Differential patterns of activation between the Ferber and Makuuchi studies were attributed to the lack of an appropriate motor control condition in the Makuuchi study [94], as well as reliance on a less complex copying paradigm. Collectively, these studies underline the importance of the left occipital and lingual gyrus in the integral functioning of the visual buffer involved in visual imagery as well as in drawing when copying line-by-line. 


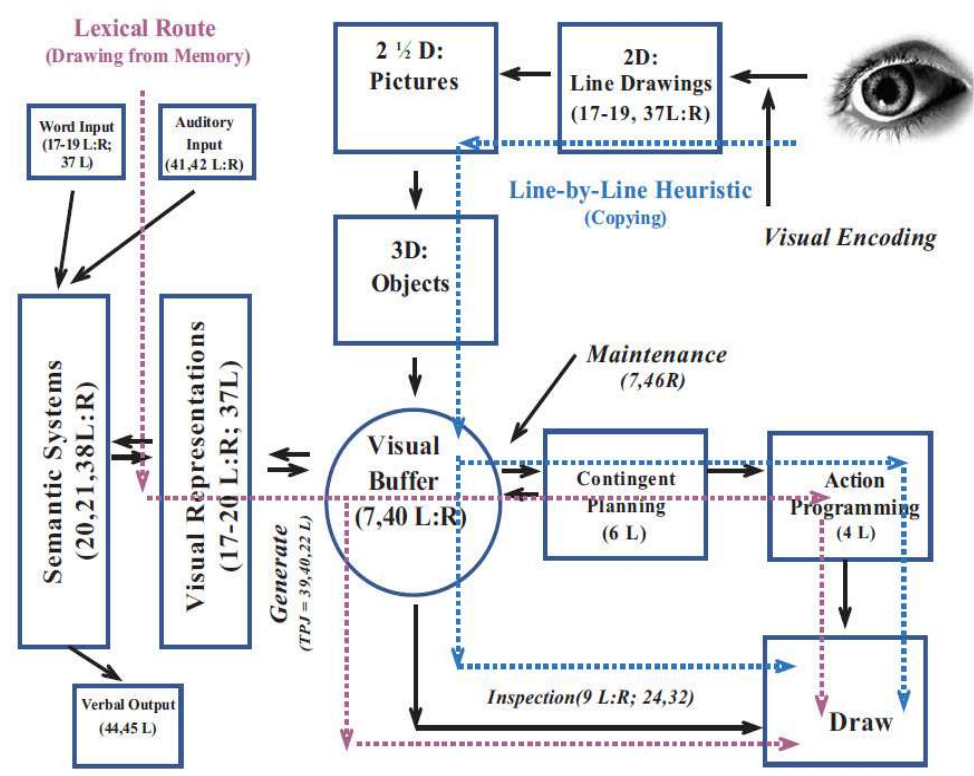

Figure 5. Hypothesized model of the functional neuroanatomy associated with free-drawing-related constructional apraxia. Numbers in parenthesis correspond to specific Brodmann's neuroanatomical regions. $17=$ primary visual cortex, $18=$ secondary visual cortex, $19=$ associative visual cortex, $20=$ inferior temporal gyrus and $37=$ fusiform gyrus involved in visual perception and imagery; $7=$ precuneus or superior parietal lobule and $40=$ supramarginal gyrus associated with the visual buffer; $20=$ inferior temporal gyrus, $21=$ middle temporal gyrus and $38=$ temporopolar region involved in high level object representations and semantic memories; 41, 42 = primary and auditory association cortex involved in auditory input; $6=$ premotor cortex involved in contingent planning based on sensory feedback; 4 = primary motor cortex involved in action programming with output via the corticospinal tracts; $9=$ dorsolateral prefrontal cortex and 24, 32 = anterior cingulate cortex involved in error monitoring associated with inspection; $7=$ superior parietal lobule and $46=$ dorsolateral prefrontal cortex involved in spatial working memory maintenance; TPJ = temporoparietal junction including: $22=$ superior temporal gyrus, 39 = angular gyrus, $40=$ supramarginal gyrus involved in generating mental images; $L=$ left hemisphere, $R=$ right hemisphere. Note that in this diagram there is an assumption of right-handedness with left lateralized language and praxis as occurs in the largest proportion of the general population.

Harrington et al. (2007) compared the neural basis for imagined writing and drawing in an fMRI study [99]. Brain areas that were activated for imagined drawing (yet were not activated for imagined writing) included the following: bilateral insular cortex (BA 13), right posterior inferior temporal cortex (BA 37), right middle and right inferior frontal cortex (BA 46/9), and the left inferior frontal cortex (BA 47). Activations associated only with actual drawing minus writing were found in the bilateral associative visual cortex (BA 19) as well as the right superior frontal gyrus (BA 9). Actual drawing minus writing would be expected to emphasize visual imagery perhaps explaining the visual associative cortex activation. The next stage of the Harrington study was to compare the two imagined conditions with actual motor-based drawing and writing. Both tasks with motor output revealed, (not surprisingly), greater activation within the left precentral gyrus (BA 4) in a sub-sample of 6 right-handed participants. Motor-based drawing as opposed to imagined drawing also revealed greater activation of left superior frontal gyrus (BA 6).

The Harrington et al. (2007) study findings are congruent with previous studies showing that mental imagery of motor processes versus actual self-generated motor processes require essentially the same motor cortices [100, 101]. The Makuuchi study did not report activation in the left posterior inferior temporal gyrus or within the anterior aspects of the inferior frontal gyrus. These brain areas are involved in naming and the Makuuchi study used naming as the baseline subtraction condition and so activation in these two regions would be expected to be cancelled out. In the Harrington et al. (2007) study only drawing resulted in activation of the anterior-most aspect of the posterior-inferior temporal gyrus and fusiform gyrus [99]. The occipitotemporal cortex strip appears to be involved in object recognition processes from simple to complex. Moore and Price (1999) divided the occipitotemporal cortex strip or BA 37 into anterior, middle, and posterior segments involved in semantic processing, naming and object feature-class processing, respectively [102]. These studies show that essentially the same area is involved in orthographic reading and visual feature extraction of objects.

Harrington et al. (2007) found that the anterior, middle, and posterior regions of BA 37 were all more active during drawing compared with writing, implying increased activation between object features and semantic processing for the drawing condition. These researchers found that right hemisphere activation within area BA 37 only extended from the posterior to middle region, but not the anterior region, which in the left rostral occipitotemporal region is involved in semantic processing [102]. Increased activation of BA 37 bilaterally for drawing may facilitate increased lexicosemantic access and activation of long-term visual memories [103] for both visual and verbal object-based codes. As Harrington et al. (2007, p. 456) note, this relationship between lexical-semantic integration and drawing was first suggested by Gainotti et al., (1983) - [64]. Collectively these studies suggest that such enhanced lexicosemantic access should be able to be distinguish neural activity between 
drawings of equivalent structural difficulty in depiction but different levels of richness of semantic associates using an fMRI adaptation paradigm [104].

However, what is the precise mechanisms associated with enhanced semantic and lexical word access facilitated by drawing in functional neuroimaging studies? In so far as the findings of the Harrington et al. (2007) study are concerned there was robust activation of the right hemisphere homologue of Broca's area [99]. There was strong bilateral activation of BA 7 or superior parietal lobule in drawing in the Harrington study and previous studies have shown that visual imagery tasks with high spatial transformation demands normally elicit bilateral activation in BA 7 [105]. The motor areas that were activated in the Harrington et al. study included the supplementary motor area, premotor gyrus, and middle frontal gyrus. These findings are expected and not new and thus could function as a verifiable "tight task" functional MRI brain-task localizer. Deiber and colleagues (1998) showed that the supplementary motor area is involved in preparation of movements [106] and a premotor focus of activation was found to be close to Exner's area which is involved in writing [107]. Contemporary views of the behavioral functions associated with Exner's area are that it is involved in planning motor images rather than being involved exclusively with writing [108].

In another imaging study, Ogawa and Inui (2009) examined the relationship between drawing by copying with activation in the posterior parietal cortex [109]. The Ogawa and Inui study illustrates some of the regions of interest involved in instantiating the line-by-line heuristic or drawing via copying pathway in the model (see Figure 4). Specifically, Ogawa and Inui found that copying versus tracing under visual or memory guidance only activated the bilateral intraparietal sulcus (BA 7, 40). These findings imply dependence on visual buffer-augmented remembrance of line drawings when transferring parts of graphics to a newer part of the screen or paper. Behavioral data similarly showed a significantly increased reaction time for copying compared with tracing with both visual feedback and memory-guided processes [109]. Since these tasks were well-equated for visuomotor and visuoperceptual functions, the findings suggest a specific time-limited spatial transformation that is required for copying. Similarly, Graziano and Gross (1998) demonstrated the involvement of the posterior parietal cortex in transforming retinal coordinates into viewer-dependent representations used for visuomotor control in monkeys [110].

The Ogawa and Inui (2009) study demonstrated bilateral intraparietal sulcus (IPS) activation (BA 7, 40) in 26 participants. Seventy-three percent demonstrated left intraparietal sulcus activation and seventy-six percent demonstrated right intraparietal sulcus activation. With availability of the visual model during copying, bilateral middle occipital gyrus activation extending into the fusiform gyri was also noted. The results imply that the bilateral intraparietal sulci are activated independent of visual or memory-guidance in copying and that these regions must therefore play a primary role in coordinate transformations
[109]. Ogawa and Inui's study used an effective motor tracing control task as the comparison for the copying condition unlike in the Makkuchi et al. (2003) study [94]. These differences in control comparison tasks may explain the greater involvement of a distributed motor network in the earlier study whereas bilateral IPS activation was the predominant finding of the Ogawa and Inui (2009) study [109]. It appeared that motor functioning requirements had been successfully subtracted in the Ogawa study whereas the ventral posterior premotor cortex (BA 44/6) was nonetheless still shown to be activated in mental image generation (see Figure 5). The results suggested that the ventral premotor cortex plays a role in rotation of mental images based on coordinate transformations occurring in the IPS.

\section{Conclusions and Future Directions}

Trojano and colleagues (2009) noted that many cognitive models of drawing are not sufficiently supported by empirical data [111]. The cognitive neuropsychological complexity of drawing processes, not to mention free-drawing's probably greater inherent complexity, increases the probability that any particular error could be a function of visuospatial, attention, spatial representations, semantic representations, motor planning and execution as well as spatial manipulation variables. Systematic attempts to characterize errors in single participants are thus scant. These authors noted that although a special issue of Cortex (2009) was devoted to the Cognitive Neurosciences of Drawing, there have been few reviews that have dealt with the representational, semantic, lexical, communicative, and symbolic facets of drawing. That issue of Cortex was exclusively focused on the motor planning basis of drawing. Hence, it appears that a broad range of neurological pathologies can result in drawing disorders through various mechanisms or disconnections of neural pathways. Progress on the lexical and semantic research front may pay higher scientific dividends in the short-term compared to purely motor-based accounts of CA, drawing and free-drawing in particular for reasons that have been explained herein. The model depicted in Figure 5 is a tentative model explicated here to stimulate further research. It is unknown whether some of the established patterns of neuropsychological findings integrated in this theoretical overview will be borne out. However hopefully it provides a better vantage point from which to order the multitude of findings of the complex disorder of constructional apraxia.

\section{References}

[1] P. Turchin, "Long-term population cycles in human societies," Ann. N.Y. Acad. Sci., vol. 1162, pp. 1-17, 2009.

[2] F. Goodenough, Measurement of Intelligence by Drawings. New York: World Books 1926.

[3] C.H. Swenson, "Empirical evaluations of human figure drawings," Psychol. Bull.,vol. 54, pp. 431-466, 1957. 
[4] C.H. Swenson, "Empirical evaluations of human figure drawings 1957-1966,” Psychol. Bull., vol. 70, pp. 20-44, 1968.

[5] S. Kahill, "Human figure drawings in adults: An update of the empirical evidence 1967-1982," Can. Psychol., vol. 25, pp. 269-292, 1984.

[6] E. Dyck, "Flashback: Psychiatric experimentation with LSD in historical perspective," Can. J. Psychiatry, vol. 50, pp. 381$388,2005$.

[7] J. Schwoebel and H.B. Coslett, "Evidence for multiple distinct representations of the human body," J. Cogn. Neurosci., vol. 17, pp. 543-553, 2005.

[8] B. Kolb and I.Q. Whishaw, Fundamentals of Human Neuropsychology - Fourth Edition, New York: W.H. Freeman and Company, 1996.

[9] J.A. Naglieri, Draw-A-Person: A Quantitative Scoring System, San Antonio, Texas: Psychological Corporation, 1998.

[10] C.R. Reynolds and J.A. Hickman, DAP: IQ: Draw-A-Person Intellectual Ability Test for Children, Adolescents and Adults, Austin, Texas: ProEd, 2004.

[11] A.B. Silverstein and G.D. Klee, "A psychopharmacological test of the body image hypothesis," J. Nerv. Ment. Dis., vol. 127, pp. 323-329, 1958.

[12] C. Savage, "Variations in ego feeling induced by d-lysergic acid diethylamide (LSD-25)," Psychoanalyt. Rev., vol. 42, pp. 1-16, 1955.

[13] R.S. Liebert, H. Werner and S. Wapner, "Studies in the effect of lysergic acid diethylamide (LSD-25): Self- and object-size perception in schizophrenics and normal adults," AMA Arch. Neurol. Psychiatry, vol. 79, pp. 580-584, 1958.

[14] S. Priebe and F. Rohricht, "Specific body image pathology in acute schizophrenia," Psychiatry Res., vol. 101, pp. 289-301, 2001.

[15] A. Levine, H.A. Abramson, M.R. Kaufman and S. Markham, "Lysergic acid diethylamide (LSD-25): XVI. The effect on intellectual functioning as measured by the Wechsler-Bellevue Intelligence Scale,” J. Psychol., vol. 40, pp. 385-395, 1955.

[16] D. Wechsler, The Wechsler-Bellevue Scale - Form II, New York: Psychological Corporation, 1946.

[17] M.E. Jarvik, H.A. Abramson and M.W. Hirsch, "Lysergic acid diethylamide (LSD-25): VI. Effect upon recall and recognition of various stimuli," J. Psychol., vol. 39, pp. 443-454, 1955.

[18] T. Passie, J.H. Halpern, D.O. Stichtenoth et al. "The pharmacology of lysergic acid diethylamide: A review," CNS Neurosci. Ther., vol. 14, pp. 295-314, 2008.

[19] The Economist. "LSD acid test: Research into hallucinogens begins to shake off decades of taboo," June 23, 2011. http://www.economist.com/node/18864332

[20] S.H. Snyder and M. Reivich, "Regional localization of LSD in monkey brain," Nature, vol. 209, pp. 1093-1095, 1966.

[21] D. De Ridder, K. Van Laere, P. Dupont, T. Menovsky and P. Van de Heyning, "Visualizing out-of-body experience in the brain,” New Eng. J. Med., vol. 357, pp. 1829-1833, 2007.

[22] D. Wechsler, The Wechsler Adult Intelligence Scale, New York: Psychological Corporation, 1955.
[23] K.A. Welsh, N. Butters, R.C. Mohs et al., "The consortium to establish a registry for Alzheimer's disease (CERAD). Part V: A normative study of the neuropsychological battery," Neurology, vol. 44, pp. 609-614, 1994.

[24] K.H. Goldstein KH and M. Scheerer M. "Tests of abstract and concrete behavior," in Contributions to Medical Psychology, vol. II, A. Weidner, Ed. New York: Ronald Press, 1953.

[25] M.D. Lezak, D.B. Howieson and D.W. Loring, Neuropsychological Assessment - Fourth edition, New York: Oxford University Press, 2004.

[26] D.F. Benson and M.I. Barton, "Disturbances in constructional ability," Cortex, vol. 6, pp. 19-46, 1970.

[27] S. Roncato, G. Sartori, J. Masterson and R. Rumiati, "Constructional apraxia: An information processing approach,” Cogn. Neuropsychol., vol. 4, pp. 113-129, 1987.

[28] A.L. Benton, "The visual retention test: A constructional praxis task," Confin. Neurol., vol. 22, pp. 141-155, 1962.

[29] E. De Renzi and L.A. Vignolo, " The token test: A sensitive test to detect receptive disturbances in aphasics," Brain, vol. 85, pp. 665-678, 1962.

[30] E.K. Warrington and A.M. Taylor, "Contribution of the right parietal lobe to object recognition," Cortex, vol. 9, pp. 152164, 1973.

[31] D. Tranel, D. Rudrauf, E.P.M. Vianna and H. Damasio, "Does the clock drawing test have focal neuroanatomical correlates?," Neuropsychology, vol. 22, pp. 553-562, 2008.

[32] E.K. Warrington, M. James and M. Kinsbourne, "Drawing disability in relation to laterality of lesion," Brain, vol. 89, pp. 53-82, 1966.

[33] J.C. Raven, "Progressive Matrices: A Perceptual Test of Intelligence - Individual Form," Oxford: Oxford University Press, 1938.

[34] L.D. Costa and H.G. Vaughn, "Performance of participants with lateralized cerebral lesions. 1: Verbal and perceptual tests,” J. Nerv. Ment. Dis., vol. 134, pp. 162-168, 1962.

[35] D. Wechsler, Wechsler Adult Intelligence Scale - Fourth Edition, San Antonio, Texas: Psychological Corporation, 2008.

[36] J. Glascher, D. Rudrauf, R. Colom et al., "Distributed neural system for general intelligence revealed by lesion mapping," $\mathrm{P}$. Natl. Acad. Sci. USA, vol. 107, pp. 4705-4709, 2010.

[37] K. Hugdahl and R. Westerhausen, The Two Halves of the Brain: Information Processing in the Cerebral Hemispheres, Cambridge, Massachusetts: MIT Press, 2010.

[38] A.R. Luria, "Higher Cortical Functions in Man, (Originally published by Moscow University Press, 1962), Translated by Basil Haigh, New York: Basic Books, 1966.

[39] J. Gottlieb and L.H. Snyder "Spatial and non-spatial functions of the parietal cortex," Curr. Opin. Neurobiol., vol. 20, pp. 731-740, 2010.

[40] A. Pascual-Leone and R. Hamilton, "The metamodal organization of the brain," Prog. Brain Res., vol. 134, pp. 427$445,2001$.

[41] A.D. Milner and M.A. Goodale, The Visual Brain in Action: Second Edition. New York: Oxford University Press, 2006. 
[42] M.A. Peterson and G. Rhodes, Perception of Faces, Objects, and Scenes: Analytic and Holistic Processes, New York: Oxford University Press, 2003.

[43] L.C. Robertson, Space, Objects, Minds and Brains, New York: Psychology Press, 2004.

[44] P. van Sommers, "A system for drawing and drawing-related neuropsychology," Cogn. Neuropsychol. Vol. 6, pp. 117-164, 1989.

[45] M. Casagrande, "Laterality and sleep," in The Two Halves of the Brain: Information Processing in the Cerebral Hemispheres, K. Hugdahl and R. Westerhausen, Eds., Cambridge, Massachusetts: MIT Press, 2010, pp. 313-345.

[46] S.M. Kossyln, J.D. Holtzman, M.J. Farah and M.S. Gazzaniga, "A computational analysis of mental image generation: Evidence from functional dissociations in split-brain participants," J. Exp. Psychol. Gen., vol. 114, pp. 311-341, 1985.

[47] M.C. Corballis and J. Sergent, "Imagery in a commissurotomised patient," Neuropsychologia, vol. 26, pp. 13-26, 1988.

[48] D. Marr, Vision, New York: Freeman, 1982.

[49] P.A. Osterreith, "Le test de copie d'une figure complexe," Arch. Psychologie, vol. 30, pp. 206-356, 1944.

[50] M.J. Farah, "The neurological basis of mental imagery: A componential analysis," Cognition, vol. 18, pp. 245-272, 1984.

[51] F. Guerin, B. Ska and S. Bellevue, "Cognitive processing of drawing abilities," Brain Cogn. vol. 40, pp. 464-478, 1999.

[52] J.L. Signoret and P. North, Les Apraxies Gestuelles, Paris: Masson, 1979.

[53] C. Semenza, G. Denes, V. D’Urso, O. Romano and T. Montorsi, "Analytic and global strategies in copying designs by unilaterally brain-damaged participants," Cortex, vol. 14, pp. 404-410, 1978.

[54] L. Trojano and D. Grossi, "A critical review of mental imagery defects," Brain Cogn. ,vol. 24, pp. 213-243, 1994.

[55] M.J. Farah, "Is visual imagery really visual? Overlooked evidence from Neuropsychology," Psychol. Rev., vol. 9., pp. 307-317, 1988.

[56] M.J. Farah, "The neuropsychology of mental imagery," in Handbook of Neuropsychology - Volume 2, F. Boller and J. Grafman, Eds. Amsterdam: Elsevier, 1989, pp. 395-414.

[57] M.J. Farah, "The neurological basis of mental imagery," in Visual Cognition, S. Pinker Ed. Cambridge, Massachusetts: MIT Press, 1985, pp. 245-271.

[58] D.N. Levine, "Prosopagnosia and visual object agnosia: A behavioral study,” Brain Lang. vol. 5, pp. 341-365, 1978.

[59] M.J. Farah, Visual Agnosia - Second Edition. Cambridge, Massachusetts: MIT Press, 2004.

[60] E. Bisiach and C. Luzzatti, "Unilateral neglect of representational space,” Cortex, vol. 14, pp. 129-133, 1978.

[61] P.W. Halligan, J.C. Marshall and D.T. Wade, "Visuospatial neglect: Underlying factors and test sensitivity," Lancet, vol. 2, pp. 908-911, 1989.
[62] K.A. Ericsson and H.A. Simon, Protocol Analysis: Verbal Reports as Data, Cambridge, Massachusetts, MIT Press, 1984.

[63] M. Critchley, The Parietal Lobes, New York: Hafner, 1953.

[64] G. Gainotti, M.C. Silveri, G. Villa and C. Caltagirone, "Drawing objects from memory in aphasia," Brain, vol. 106, pp. 613-622, 1983.

[65] G. Goldenberg and C. Artner, "Visual imagery and knowledge about the visual appearance of objects in participants with posterior cerebral artery lesions," Brain Cogn., vol. 15, pp. 160-186, 1991.

[66] D. Dupuy and O. Godefroy, "Disorders of visuoconstructive ability," in The Behavioral and Cognitive Neurology of Stroke, O. Godefroy and J. Bogousslavsky, Eds. Cambridge, Massachusetts: Cambridge University Press, 2007, pp. 254-268.

[67] G. Gainotti, "Constructional apraxia," in Handbook of Clinical Neurology - Volume 1, J.A.M. Frederiks, Ed. Amsterdam: Elsevier, 1985; pp. 491-506.

[68] R. Arena and G. Gainotti, "Constructional apraxia and visuoperceptive disabilities in relation to laterality of cerebral lesions," Cortex, vol. 14, pp. 463-473, 1978.

[69] G. Gainotti, "A quantitative study of the "closing-in' behavior symptom in children and in brain damaged participants," Neuropsychologia, vol. 10, pp. 429-436, 1972.

[70] K. Heilman and E. Valenstein, Eds. Clinical Neuropsychology - Fourth Edition. New York: Oxford University Press, 2003.

[71] H. Nielsen, "Is constructional apraxia primarily an interhemispheric disconnection syndrome?," Scand. J. Psychol., vol. 16, pp. 113-124, 1975.

[72] A. Kirk and A. Kertesz, "Hemispheric contributions to drawing," Neuropsychologia, vol. 27, pp. 881-886, 1989. .

[73] L . Trojano and M. Conson, " Visuospatial and visuoconstructive deficits," in Handbook of Clinical Neurology - Volume 88: Neuropsychology and Behavioral Neurology, G. Goldenberg and B.L. Miller, Eds. Amsterdam, Elsevier, 2008; pp. 373-391.

[74] A.R. Luria and L.S. Tsvetkova, "The programming of constructive ability in local brain injury," Neuropsychologia, vol. 2, pp. 95-108, 1964.

[75] J. Dérouesné, X. Seron and J.L. Signoret, "La rééducation de participants with lésion frontale," Revue Neurol. (France), vol. 131, pp. 677-689, 1975.

[76] B. Pillon, "Troubles visuoconstructifs et méthods de compensation: Résultats de 85 participants atteints de lésions cérebrales," Neuropsychologia, vol. 19, pp. 375-383, 1981.

[77] M.F. Folstein, S.E. Folstein and P.R. McHugh, "Mini-mental state: A practical method for grading the cognitive state of patients for the clinician," J. Psychiat. Res. Vol. 12, pp. 189-198, 1975. .

[78] G. Villa, G. Gainotti and C. De Bonis, "Constructive disabilities in focal brain-damaged participants: Influence of hemispheric side, locus of lesion and co-existent mental deterioration," Neuropsychologia, vol. 25, pp. 497-510, 1986.

[79] L. Trojano, N.A. Fragassi, L. Chiacchio et al. "Relationships between constructional and visuospatial abilities in normal participants and in focal brain-damaged participants," J. Clin. Exp. Neuropsychol., vol. 26, pp. 1103-1112, 2004. 
[80] E. De Renzi, Disorders of Space Exploration and Cognition, New York: Wiley and Sons, 1982.

[81] F.W. Black and B.A. Bernard, "Constructional apraxia as a function of lesion locus and size in participants with focal brain damage," Cortex, vol. 20, pp. 111-120, 1984.

[82] R.S. Marshall, R.M. Lazar, J.R. Binder et al. "Intrahemispheric localization of drawing dysfunction," Neuropsychologia, vol. 32, pp. 493-501, 1994.

[83] D. Grossi, La Riabilitazione dei Disturbi della Cognizione Spaziale, Milano: Masson, 1991.

[84] L. Trojano and D. Grossi, "Pure constructional apraxia - A cognitive analysis of a single case," Behav. Neurol., vol. 11, pp. 43-49, 1998.

[85] F. Tam, N.W. Churchill, S.C. Strother and S.J. Graham, "A new tablet for writing and drawing during functional MRI," Hum. Brain Mapp., vol. 32, pp. 240-248, 2011.

[86] L.A. Hayduk, Structural Equation Modeling with LISREL: Essentials and Advances. Baltimore, Maryland: John Hopkins University Press, 1987.

[87] M.S. Gazzaniga, J.E. Bogen and R.W. Sperry, "Observations on visual perception after disconnexion of the cerebral hemispheres in man,” Brain, vol. 88, pp. 221-236, 1965.

[88] J.E. LeDoux, D.H. Wilson and M.S. Gazzaniga, "Manipulospatial aspects of cerebral lateralization: Clues to the origin of lateralization," Neuropsychologia, vol. 15, pp. 743-750, 1977.

[89] A. Kirk and A. Kertesz, "Subcortical contributions to drawing,". Brain Cogn. vol. 21, pp. 51-70, 1993.

[90] A. Kertesz, The Western Aphasia Battery, New York: Grune and Stratton, 1982.

[91] B. Laeng, "Constructional apraxia after left or right unilateral stroke,” Neuropsychologia, vol. 44, pp. 1595-1606, 2006.

[92] S.M. Kosslyn, C.F. Chabris, C.J. Marsolek and O. Koenig, "Categorical versus coordinate spatial relations: Computational analyses and computer simulations," J. Exp. Psychol. Hum. Percept. Perform., vol. 19, pp. 562-577, 1992.

[93] G. Jager and A. Postma, "On the hemispheric specialization for categorical and coordinate spatial relations: a review of the current evidence," Neuropsychologia, vol. 41, pp. 504-515, 2003.

[94] M. Makuuchi, T. Kaminaga and M. Sugishita, "Both parietal lobes are involved in drawing: A functional MRI study and implications for constructional apraxia," Cogn. Brain. Res., vol. 16, pp. 338-347, 2003.

[95] D. Farias, C. Davis and G. Harrington, "Drawing: Its contribution to naming in aphasia," Brain Lang., vol. 97, pp. 53-63, 2006.

[96] A. Martin, J.V. Haxby, F.M. Lalonde et al. "Discrete cortical regions associated with knowledge of color and knowledge of action," Science, vol. 270, pp. 102-105, 1995.
[97] R.A Poldrack, A.D. Wagner, M.W. Prull et al., "Functional specialization for semantic and phonological processing in the left inferior prefrontal cortex," NeuroImage, vol. 10, pp. 15-35, 1999.

[98] S. Ferber, R. Mraz, N. Baker and S.J. Graham, "Shared and differential neural substrates of copying and drawings: A functional magnetic resonance imaging study," NeuroReport, vol. 18, pp. 1089-1093, 2007.

[99] G.S. Harrington, D. Farias, C.H. Davis and M.H. Buonocore, "Comparison of the neural basis for imagined writing and drawing," Hum. Brain Mapp., vol. 28, pp. 450-459, 2007.

[100] J. Decety, "Do imagined and executed actions share the same neural substrate?," Brain Res. Cogn. Brain Res., vol. 3, pp. 8793,1996.

[101] C. Stippich, H. Ochmann and K. Sartor, "Somatotopic mapping of the human primary sensorimotor cortex during motor imagery and motor execution by functional magnetic resonance imaging," Neurosci. Lett., vol. 331, pp. 50-54, 2002

[102] C.J. Moore and C.J. Price, "Three distinct ventral occipitotemporal regions for reading and object naming," NeuroImage, vol. 10, pp. 181-192, 1999.

[103] K. Usui, A. Ikeda, M. Takayama et al. "Conversion of semantic information into phonological representation: A function of left posterior basal temporal area," Brain, vol. 126, pp. 632-641, 2003.

[104] K. Grill-Spector and R. Malach, "fMR-adaptation: a tool for studying the functional properties of human cortical neurons," Acta Psychol. (Amsterdam), vol. 107, pp. 293-321, 2001.

[105] S.M. Kossyln, G. Ganis and W.L. Thompson, "Neural foundations of imagery," Nat. Rev. Neurosci. vol. 2, pp. 635$642,2001$.

[106] M.P. Deiber, V. Ibanez, M. Honda et al. "Cerebral processes related to visuomotor imagery and generation of simple finger movements studied with positron emission tomography," NeuroImage, vol. 7, pp. 73-85, 1998.

[107] S. Exner, Untersuchungen uber die Localisation der Functionen in der Grosshirnrinde des Menschen, Wien: W. Braumuller, 1881.

[108] M. Longcamp, J.L. Anton, M. Roth and J.L. Velay, "Visual presentation of single letters activates a premotor area involved in writing," NeuroImage, vol. 19, pp. 1492-1500, 2003.

[109] K. Ogawa and T. Inui, "The role of the posterior parietal cortex in drawing by copying," Neuropsychologia, vol. 47, pp. 1013-1022, 2009.

[110] M.S. Graziano and C.G. Gross, "Spatial maps for the control of movement," Curr. Opin. Neurobiol., vol. 8, pp. 195-201, 1998.

[111] L. Trojano, D. Grossi and T. Flash, "Cognitive neuroscience of drawing: Contributions of neuropsychological, experimental and neurofunctional studies," Cortex, vol. 45, pp. 269-277, 2009. 